This item was submitted to Loughborough's Research Repository by the author.

Items in Figshare are protected by copyright, with all rights reserved, unless otherwise indicated.

\title{
Cold water ingestion improves exercise tolerance of heat-sensitive people with MS
}

PLEASE CITE THE PUBLISHED VERSION

https://doi.org/10.1249/MSS.0000000000001496

\section{PUBLISHER}

(c) American College of Sports Medicine. Published by Wolters Kluwer

\section{VERSION}

AM (Accepted Manuscript)

\section{PUBLISHER STATEMENT}

This work is made available according to the conditions of the Creative Commons Attribution-NonCommercialNoDerivatives 4.0 International (CC BY-NC-ND 4.0) licence. Full details of this licence are available at: https://creativecommons.org/licenses/by-nc-nd/4.0/

\section{LICENCE}

CC BY-NC-ND 4.0

\section{REPOSITORY RECORD}

Chaseling, Georgia K., Davide Filingeri, Michael Barnett, Phu Hoang, Scott L. Davis, and Oliver E. Jay. 2019. "Cold Water Ingestion Improves Exercise Tolerance of Heat-sensitive People with MS". figshare. https://hdl.handle.net/2134/33837. 
Cold water ingestion improves exercise tolerance of heat-sensitive people with MS

\author{
Georgia K Chaseling ${ }^{1}$, Davide Filingeri ${ }^{1,2}$, Michael Barnett ${ }^{3}$, \\ Phu Hoang ${ }^{4}$, Scott L Davis ${ }^{5,6}$, Ollie Jay ${ }^{1,7} \bowtie$
}

\begin{abstract}
${ }^{1}$ Thermal Ergonomics Laboratory, Faculty of Health Sciences, University of Sydney, Lidcombe NSW, Australia

${ }^{2}$ Environmental Ergonomics Research Centre, Loughborough University, Leicestershire, United Kingdom

${ }^{3}$ Brain and Mind Research Centre, Sydney Medical School, University of Sydney, Camperdown, NSW, Australia

${ }^{4}$ Neuroscience Research Australia, Sydney, NSW, Australia

${ }^{5}$ Applied Physiology \& Wellness Southern Methodist University, Dallas, TX, United States

${ }^{6}$ Neurology and Neurotherapeutics, University of Texas Southwestern Medical Center, Dallas, TX, United States

${ }^{7}$ Charles Perkins Centre, University of Sydney, Camperdown, NSW, Australia
\end{abstract}

Short title: Cold drinks, Exercise and Multiple Sclerosis

\title{
Address for correspondence:
}

Dr. O. Jay $₫$

Thermal Ergonomics Laboratory,

Faculty of Health Sciences,

University of Sydney,

Lidcombe, NSW, 2141

AUSTRALIA

Tel: +61 293519328

Fax: +61 293519204

E-mail: ollie.jay@sydney.edu.au 


\section{Abstract}

2 Purpose: Heat intolerance commonly affects the exercise capacity of people with multiple

3 sclerosis (MS) during bouts of hot weather. Cold-water ingestion is a simple cooling

$4 \quad$ strategy but its efficacy for prolonging exercise capacity with MS remains undetermined.

5 We sought to identify whether cold-water ingestion blunts exercise-induced rises in body

6 temperature and improves exercise tolerance in heat-sensitive individuals with MS.

7 Methods: On two separate occasions, 20 participants (10 relapsing-remitting MS (EDSS: 1-

8 5); 10 age- and fitness-matched healthy controls (CON)) cycled at $40 \% \mathrm{VO}_{2} \mathrm{max}$ at $30^{\circ} \mathrm{C}$,

$930 \%$ RH until volitional exhaustion (or a maximum of $60 \mathrm{~min}$ ). Every 15 minutes, participants ingested $3.2 \mathrm{~mL} \cdot \mathrm{kg}^{-1}$ of either $1.5^{\circ} \mathrm{C}(\mathrm{CLD})$ or $37^{\circ} \mathrm{C}(\mathrm{NEU})$ water. Rectal $\left(T_{\mathrm{re}}\right)$ temperature, mean skin ( $\left.T_{\mathrm{sk}}\right)$ temperature, and heart rate $(\mathrm{HR})$ were measured throughout.

12 Results: All $10 \mathrm{CON}$, but only 3 of 10 MS participants completed 60 minutes of exercise in

13 NEU trial. The remaining 7 MS participants all cycled longer $(\mathrm{P}=0.006)$ in CLD

14 (46.4 $\pm 14.2 \mathrm{~min})$ compared to $\mathrm{NEU}(32.7 \pm 11.5 \mathrm{~min})$, despite similar elevations in absolute $15 T_{\text {re }}\left(\mathrm{NEU}: 37.32 \pm 0.34^{\circ} \mathrm{C}\right.$; CLD: $\left.37.28 \pm 0.26^{\circ} \mathrm{C} ; \mathrm{P}=0.44\right)$, change in $T_{\text {re }}\left(\mathrm{NEU}: 0.38 \pm 0.21^{\circ} \mathrm{C}\right.$;

16 CLD: $0.34 \pm 0.24^{\circ} \mathrm{C}$ ), absolute $T_{\text {sk }}\left(\mathrm{NEU}: 34.48 \pm 0.47^{\circ} \mathrm{C} ; \mathrm{CLD}: 34.44 \pm 0.54^{\circ} \mathrm{C} ; \mathrm{P}=0.82\right.$ ) and

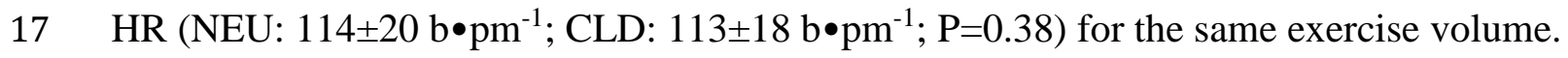

18 Conclusions: Cold-water ingestion enhanced exercise tolerance of MS participants in the

19 heat by $\sim 30 \%$ despite no differences in core and mean skin temperatures or heart rate.

20 These findings support the use of a simple cooling strategy for mitigating heat intolerance

21 with MS, and lend insight into the potential role of cold-afferent thermoreceptors that

22 reside in the abdomen and oral cavity in the modulation of exercise tolerance with MS in 23 the heat. 
24 Keywords: Uhthoff's phenomenon, fatigue, physical activity, heat sensitivities. 


\section{Introduction}

It is well documented that during physical activity and/or exposure to hot environments individuals with multiple sclerosis (MS) can experience heat intolerance (1), which is typically characterized by a rapid onset of fatigue (2). Despite its prevalence, the underlying mechanisms responsible for this phenomenon (Uhthoff's) remain somewhat inconclusive. Nevertheless, since the work by Davis (3) and Rasminsky (4) it has been generally considered that a rise in core temperature of $\sim 0.5^{\circ} \mathrm{C}$ induces heat-related fatigue secondary to slowed or blocked conduction of demyelinated nerves. As such, people with MS are regularly advised to remain indoors during hot weather, and limit physical activity, which can substantially impact employability and/or quality of life (5).

\section{Some cooling strategies administered before and/or during heat exposure} successfully mitigate the development of heat-related fatigue in people with MS (6). However, these methods, such as 30 minutes of lower body cold water immersion (7) or donning an ice vest (8) can prove impractical in the context of everyday life and incompatible with many jobs. Cold fluid ingestion during physical activity is a simple strategy that is presently recommended by, among others, the National MS Society (9) the MS Society (UK) (10) and MS Queensland (Australia). Indeed, drinking cold water could effectively mitigate elevations in core temperature and associated fatigue as it introduces an internal heat loss avenue (via conduction) in addition to evaporative and convective heat loss from the skin surface. Nevertheless, to the best of our knowledge, no study has yet assessed whether cold fluid ingestion during exercise in the heat can mitigate rises in core temperature and accompanying fatigue in people with MS. 
48 to thermoneutral $\left(37^{\circ} \mathrm{C}\right)$ water on exercise time to exhaustion at a fixed low relative

49 intensity ( $\left.\sim 40 \% \mathrm{VO}_{2 \mathrm{max}}\right)$, and the elevation in core temperature of heat-sensitive relapsing-

50 remitting MS participants in a warm $\left(30^{\circ} \mathrm{C}\right)$ environment. It was hypothesized that with

51 thermoneutral water ingestion, exercise time to exhaustion would be shorter for MS

52 compared to age- and fitness-matched control participants. It was also hypothesized that

53 compared to thermoneutral water ingestion exercise time to exhaustion in MS participants

54 would be extended with cold water ingestion due to a blunted rise in core temperature. 


\section{Methods}

Participants

7

8
disability status scale (EDSS) range of 2-4.5 (1 = No disability, slight dysfunction in one area, 4.5 = significant disability with some limitation of daily activities (11)) and 10 age, height and weight-matched healthy controls (Table 1) were recruited for this study based on a power calculation (Heinrich-Heine-Universität Düsseldorf, Germany) employing an $\alpha$ of 0.05 , a $1-\beta$ of 0.95 and an effect size of 1.55 for the main outcome variable of exercise performance with cold fluid ingestion in the heat (12). All MS participants had a selfreported intolerance to the heat. All participants were informed of any risks associated with the study before providing written informed consent. The study was approved by the University of Sydney Human Research Ethics Committee (HREC No: 2016/214).

\section{Measurements}

Rectal $\left(T_{\text {re }}\right)$ temperature was measured using a general-purpose pediatric thermistor (TM400, Covidien, Mansfield, MA, USA) self-inserted to a depth of $12 \mathrm{~cm}$ past the anal sphincter. Skin temperature was measured at four sites on the right side using thermistors (Concept Engineering, Old Saybrook, CT, USA) attached with hypoallergenic tape (Blenderm, 3M, Sydney, NSW, Australia). Mean skin temperature $\left(T_{\mathrm{sk}}\right)$ was estimated using a weighted average in accordance to Ramanathan (13). All thermometric measurements were sampled at 5 seconds intervals (NI cDAQ-91722 module, National Instruments, Austin, TX, USA) and displayed in real-time using LabView (v7.0). Heart rate (HR) was measured using a wireless 6-lead ECG (Quark T12x Asia Pacific PTY, Sydney, NSW, Australia) monitoring system. Electromagnetic gel was 
applied to 4 foam electrodes, which were then placed under the right and left clavicle, the right and left $6^{\text {th }}$ intercostal and then covered with tape. Prior to the placement of the electrodes, the skin surface was shaved and cleaned with alcohol to ensure minimal signal interference.

\section{Protocol}

Each participant completed one preliminary trial and two experimental trials.

During the preliminary trial, participants performed an incremental submaximal exercise protocol (beginning at $45 \mathrm{~W}$ increasing $20 \mathrm{~W}$ every three minutes for a total of four stages) on a semi-recumbent cycle ergometer (Corival Recumbent, Lode BV, Groningen, Netherlands) in a $20^{\circ} \mathrm{C}$ room. Heart rate and oxygen consumption (Quark CPET, Cosmed, Asia Pacific PTY, Sydney, NSW, Australia) were measured during each 3-min stage. A least square regression equation was employed using sub-maximal heart rate and oxygen consumption at the end of each stage and extrapolated to the maximum age-predicated heart rate (220-age) (14) to determine $\mathrm{VO}_{2 \max }$ using the YMCA protocol (15). Individualized workloads ( $40 \%$ of predicted $\mathrm{VO}_{2 \max }$ ) were calculated for the subsequent experimental trials.

Participants completed two experimental trials separated by a minimum of $48 \mathrm{~h}$ in a climate-controlled chamber at $30^{\circ} \mathrm{C}$ and $30 \%$ relative humidity until i) volitional exhaustion, or ii) a maximum of 60 minutes. Participants were required to complete both trials at the same time of day to avoid any disparity in resting core temperature due to circadian rhythm. If any participant presented with a resting $T_{\text {re }}$ more than $0.2^{\circ} \mathrm{C}$ away from the other trials, then the trial would not commence. Participants cycled on a semirecumbent cycle ergometer at a fixed relative intensity $\left(\sim 40 \% \mathrm{VO}_{2 \max }\right)$ and consumed a 3.2 
$101 \mathrm{ml} \cdot \mathrm{kg}^{-1}$ aliquot of water (in $<1$ minute) after the $15^{\text {th }}, 30^{\text {th }}$ and $45^{\text {th }}$ minute of exercise.

102 Participants consumed either thermoneutral $\left(37^{\circ} \mathrm{C}\right)$ water $(\mathrm{NEU})$ or cold $\left(1.5^{\circ} \mathrm{C}\right)$ water

103 (CLD) during each experimental trial. The presentation of trials was balanced between

104 participants. The temperature of the water ingested in the NEU trial was maintained using

105 a hydrostatic controlled water bath (DA05A, Polyscience, Niles, IL, USA). The

106 temperature of the water ingested in the CLD trial was maintained in a thermos filled with

107 ice. Immediately prior to fluid ingestion, the temperature of the fluid was verified using a

108 factory-calibrated glass precision thermometer (Durac Plus, Blue Spirit, Cole-Parmer,

109 Vernon Hills, IL, USA) with a certified range between $-1^{\circ} \mathrm{C}$ and $+100^{\circ} \mathrm{C}$ and with an

110 accuracy of $\pm 0.1^{\circ} \mathrm{C}$, and the required mass of water was measured using a balance with a

111 precision of $0.1 \mathrm{~g}$ (MS12001L, Mettler Toledo, Columbus, OH, USA). Breath by breath

112 oxygen consumption was continuously monitored to ensure participants were performing

113 at the same relative intensity throughout both trials.

114 Statistical Analysis

115 A two-way mixed ANOVA employing the repeated factor of water temperature

116 (CLD, NEU) and the non-repeated factor of group (MS, Control) was used to examine

117 exercise time to exhaustion. The $T_{\mathrm{re}}, T_{\mathrm{sk}}$ and HR at the time of exhaustion in the shortest

118 trial for each individual were also compared to the same time point in the other trial within

119 the MS and CON groups using paired sample t-tests. A within-group analysis was employed

120 for measures of $\mathrm{T}_{\mathrm{re}}$ and $\mathrm{T}_{\mathrm{sk}}$ due to the different exercise time between the con and MS groups.

121 Furthermore, within the CLD trial $T_{\mathrm{re}}$ and $T_{\mathrm{sk}}$ at the time of exhaustion in the NEU was

122 compared to the end-exercise values of the CLD within the MS group using a paired

123 sample t-test. Finally, an unpaired t-test was used to examine HR between control and MS 
124 participants at 30 minutes of exercise for both the NEU and CLD trials...All statistical 125 analyses were performed using GraphPad Prism (v6.0, LA Jolla, CA, USA).

126 
127

128

129

130

131

132

133

134

136

138

139

140

141

142

143

144

145

146

147

148

\section{Results}

Exercise time to exhaustion was shorter in the MS group compared to the CON group $(\mathrm{P}=0.002)$, however an interaction was observed between water temperature and group $(\mathrm{P}<0.001)$. Specifically, all 10 control participants completed 60 minutes of exercise in both the NEU and CLD trials (Figure 1). On the other hand, while only 3 of 10 participants in the MS group completed 60 minutes of exercise in the NEU and CLD trial, all 7 MS participants who could not complete the NEU trial cycled longer (Figure 1) in the CLD trial (NEU: $32.7 \pm 11.5$ min; CLD: $46.4 \pm 14.2$ min; $\mathrm{P}=0.006)$. After 30 minutes of exercise, HR responses in the NEU trial (MS: $104 \pm 15 \mathrm{~b} \bullet \mathrm{pm}^{-1}$; CON: $96 \pm 10 \mathrm{~b} \bullet \mathrm{pm}^{-1}$; $\mathrm{P}=0.22$ ) and the CLD trial (MS: $103 \pm 17 \mathrm{~b} \bullet \mathrm{pm}^{-1}$; CON: $92 \pm 12 \mathrm{~b} \bullet \mathrm{pm}^{-1} ; \mathrm{P}=0.17$ ) were not different, despite being moderately higher for the MS group throughout exercise.

In the MS group, at the time of exhaustion in the NEU trial, $T_{\text {re }}(\mathrm{P}=0.44$; Figure 2A), $T_{\text {sk }}\left(\mathrm{P}=0.82\right.$; Figure 2C) , change in $T_{\text {re }}(\mathrm{P}=0.66$; Figure $2 \mathrm{E})$ and HR (NEU: $114 \pm 19$ $\mathrm{b} \bullet \mathrm{pm}^{-1}$; CLD: $113 \pm 17 \mathrm{~b} \bullet \mathrm{pm}^{-1}$; $\left.\mathrm{P}=0.45\right)$ were not different after the same amount of exercise time elapsed in the CLD trial. All 7 MS participants who cycled for longer in the CLD trial did so despite $T_{\mathrm{re}}(\mathrm{P}=0.001)$ and $T_{\mathrm{sk}}(\mathrm{P}=0.03)$ rising to higher values above baseline when they did stop exercise $\left(\Delta \mathrm{T}_{\mathrm{re}}: 0.26 \pm 0.12^{\circ} \mathrm{C}\right.$ vs. $0.40 \pm 0.23^{\circ} \mathrm{C} ; \Delta \mathrm{T}_{\mathrm{sk}}$ :

$1.27 \pm 0.72^{\circ} \mathrm{C}$ vs. $\left.1.47 \pm 0.79^{\circ} \mathrm{C}\right)$. In the CON group, end-exercise (i.e. after $60 \mathrm{~min}$ in all CON participants) $T_{\mathrm{re}}$ ( $\mathrm{P}=0.25$; Figure 2B), $T_{\text {sk }}(\mathrm{P}=0.33$; Figure $2 \mathrm{D})$, change in $T_{\mathrm{re}}(\mathrm{P}=0.7$; Figure $2 \mathrm{~F})$ and $\mathrm{HR}$ (NEU: 99 \pm 11 bpm; CLD: 99 $\pm 13 ; \mathrm{P}=0.33$ ) were not different between the NEU and CLD trial. Due to equipment problems, HR values are only displayed for 8 control participants. 


\section{Discussion}

151

152

This study is the first to report the efficacy of cold-water ingestion for improving exercise tolerance in the heat in people with MS. Importantly, all MS participants that could not complete 60-min of exercise with the ingestion of thermoneutral water (NEU trial) due to volitional exhaustion, cycled for longer with ingestion of cold water (CLD trial). However this longer exercise time to exhaustion in the CLD trial in the MS group was observed despite no influence of a lower ingested water temperature on core and skin temperatures as well as heart rate.

It is well documented that even small increases in body temperature are associated with a transient worsening of symptoms for individuals with MS (3, 4), otherwise known as Uhthoff's phenomenon (16). The development of fatigue, manifested by sensations of tiredness, is a common characteristic associated with Uhthoff's phenomenon and explains the shorter exercise time for 7 of the 10 MS who could not complete 60 minutes of exercise compared to CON group in the NEU trial as both participant groups were matched for age and aerobic fitness. In addition, the relative exercise intensity was moderately low ( $\left.\sim 40 \% \mathrm{VO}_{2 \max }\right)$ and should have been easily sustainable for 60 minutes, as evidenced by all 10 CON participants completing the exercise bout in both trials.

\section{Within the MS group, the longer exercise to time to exhaustion in the CLD trial} occurred despite a similar $\mathrm{T}_{\mathrm{re}}, \mathrm{T}_{\mathrm{sk}}$, and $\mathrm{HR}$ at a comparable time point (i.e. same volume of exercise) than the time to exhaustion in the NEU trial for each individual. In other words, exercise tolerance in the heat was improved in the MS group with cold-water ingestion despite no independent influence of ingested water temperature on the development of thermal and cardiovascular strain with exercise time. Indeed, from the time point at which 
173 exercise exhaustion was reached in the NEU trial, $\mathrm{T}_{\mathrm{re}}$ and $\mathrm{T}_{\text {sk }}$ in the CLD trial continued to

174 rise to higher values by the time exercise stopped. It has been previously suggested that the

175 underlying mechanism responsible for heat-related reduction in exercise performance in

176 healthy athletes is potentially similar to heat sensitivity with MS, but with fatigue onset

177 occurring alongside much smaller rises in body temperature with MS (17). It follows that

178 heat-related decrements in the aerobic performance of healthy athletes can potentially be

179 attenuated via the stimulation of cold-afferent receptors located in the oral cavity (12) and

180 on the skin surface (18), without necessarily lowering core temperature. The present

181 findings potentially support the notion that research examining the mitigation of heat-

182 related decrements in exercise performance in healthy athletes may, at least to an extent, be

183 translatable to the management of Uhthoff's phenomenon in the MS population.

184 Irrespective of participant group, for the same volume of exercise, any alteration in

185 core and skin temperature due to theingested fluid temperature were minimal (Fig. 2A, C

186 and E), despite the greater internal heat loss via conduction with cold fluid ingestion. A

187 recent series of studies (19-21) described fluid temperature-dependent alterations in

188 sweating during exercise that are modulated, independently of core and skin temperatures,

189 by visceral thermoreceptors located in the abdomen. Ultimately, the reduction in

190 evaporative heat loss from the skin surface with cold fluid ingestion was found to

191 counterbalance the greater internal heat loss, thereby yielding similar changes in whole

192 body heat storage and thus similar changes in core temperature, irrespective of ingested

193 fluid temperature (19). Although sweating rates are not reported in the present study, a

194 similar fluid temperature-dependent modulation of skin surface evaporation could explain

195 the similar levels of thermal strain between the NEU and CLD trials within both the MS 
and CON group. Another consideration is that the absolute amount of heat transfer

197 generated by each $3.2 \mathrm{ml} \cdot \mathrm{kg}^{-1}$ aliquot of $1.5^{\circ} \mathrm{C}$ water, even without any parallel alterations

198 of skin surface evaporation, would only be $\sim 35 \mathrm{~kJ}$, which for a $82.5 \mathrm{~kg}$ individual with a

mean body specific heat of $3.49 \mathrm{~kJ} \cdot \mathrm{kg}^{-1} \cdot{ }^{\circ} \mathrm{C}^{-1}$ would yield a reduction in mean body

temperature of only $\sim 0.1^{\circ} \mathrm{C}$.

Despite the profound impact of regular exercise on the physical and psychological

health of individuals with MS (22), it has been reported that people with MS are less

physically active (23), partly to avoid a temporary worsening of symptoms associated with

204 an elevation in body temperature. Moreover, heat intolerance has been shown to greatly

impact the capacity for many people with MS to remain among the workforce (5). Cold

water ingestion is a simple strategy for improving exercise tolerance in the heat, which

could be used as an alternative to other less practical but currently recommended cooling

strategies such as partial immersion in cold water prior to heat exposure (7), or donning an

ice vest (24). It should be noted that for individuals with MS susceptible to urinary

incontinence, additional fluid ingestion might not prove an optimal solution. Therefore,

211 future research must establish whether independently stimulating cold-afferent

212 thermoreceptors in the oral cavity, via a cold mouth rinse, would be sufficient to mitigate

213 heat-related decrements in exercise tolerance with MS, as reported with complete cold-

214 water ingestion in the present study.

\section{Limitations}

The present study does not include subjective measures such as whole body thermal sensation (WBTS) or rate of perceived exertion (RPE). If a lower WBTS and RPE were

218 observed for the same exercise load for the MS participants, this may have explained the 
219 improved exercise tolerance. Furthermore, there was no measure taken to assess the onset

220 of symptoms, if any, for the MS participants. Therefore, we are unsure whether cold fluid

221 ingestion mitigated the onset of heat related symptoms, or temporarily dampened

222 sensations of heat intolerance. Future research should investigate whether prolonged

223 exercise time effects heat related symptoms, or whether ingesting cold water is sufficient to

224 mitigate the onset of symptoms during exercise in a hot environment. As some participants

225 reported a mild discomfort with the cold fluid ingestion future research should assess

226 similar outcome variables to this current research, however with slightly warmer fluid 227 temperatures.

The exercise time to exhaustion protocol was selected to assess the capacity of an easily fatigued, non-athletic population. However, due to the large variability that is typically demonstrated in time to exhaustion studies, future research should look at

231 investigating aerobic performance for MS individuals using a more reliable protocol such 232 as a time trial (25) Using heart rate to predict $\mathrm{VO}_{2 \max }$ for MS individuals has not yet been 233 validated for this population. Given that heart rate responses to exercise were quite variable 234 for the MS group, there is a possibility that $\mathrm{VO}_{2 \max }$ results were either under or 235 overestimated. Despite this, the relative workload was consistent within each participant, 236 and therefore the main outcome of this study is not affected.

\section{Conclusion}

238 In conclusion, the present study examined the influence of ingesting cold compared 239 to thermoneutral water on exercise time to exhaustion at a fixed low relative intensity 240 ( $\left.\sim 40 \% \mathrm{VO}_{2 \max }\right)$, and the concurrent elevation in core and skin temperature of heat-sensitive 241 relapsing-remitting MS participants in a warm $\left(30^{\circ} \mathrm{C}\right)$ environment. With thermoneutral 
242 water ingestion, exercise time to exhaustion was shorter in the MS group compared to age-

243 matched controls, presumably due to the development of fatigue associated with Uhthoff's

244 phenomenon. Cold-water ingestion resulted in a 30\% longer exercise time to exhaustion

245 in the MS participants that could not complete 60 minutes of exercise in the thermoneutral

246 water ingestion trial. However while cold-water ingestion appeared to improve the exercise

247 tolerance of the MS group in the heat, it did not blunt the rise in core and mean skin

248 temperature with time. These findings provide a practical and simple cooling strategy for

249 individuals with MS performing physical activity in hot environments, and lend insight

250 into the potential role of cold-afferent thermoreceptors that reside in the abdomen and oral

251 cavity in the modulation of exercise tolerance with MS in the heat. 


\section{Acknowledgements}

254 This research was supported by a Multiple Sclerosis Research Australia Incubator 255 Grant (Grant holders: Jay, Davis, Barnett and Hoang; grant number \#14-009); a Multiple 256 Sclerosis Research Australia Postgraduate Fellowship (Grant holders: Chaseling and Jay; 257 grant number \#15-087); and an Australian Government, Department of Education, 258 Endeavour Post-Doctoral Research Fellowship (Grant holder: Filingeri). All results in this 259 study are presented clearly, honestly and without fabrication, falsification or inappropriate 260 data manipulation. Results of the present study do not constitute endorsement by the 261 American College of Sports Medicine.

262

263 Conflicts of Interest

264 There are no conflicts of interest to declare. 


\section{References}

266 1.Nelson DA, McDowell F. The effects of induced hyperthermia on patients with multiple

267 sclerosis. Journal of neurology, neurosurgery, and psychiatry. 1959;22(2):113.

268 2.Simmons RD, Ponsonby A-L, Van Der Mei IA, Sheridan P. What affects your MS? Responses to 269 an anonymous, Internet-based epidemiological survey. Multiple Sclerosis. 2004;10(2):202-11.

270 3.Davis FA, Jacobson S. Altered thermal sensitivity in injured and demyelinated nerve A possible

271 model of temperature effects in multiple sclerosis. Journal of Neurology, Neurosurgery \&

272 Psychiatry. 1971;34(5):551-61.

273 4.Rasminsky M. The effects of temperature on conduction in demyelinated single nerve fibers.

274 Archives of Neurology. 1973;28(5):287-92.

275 5.Julian LJ, Vella L, Vollmer T, Hadjimichael O, Mohr DC. Employment in multiple sclerosis. 276 Journal of neurology. 2008;255(9):1354-60.

277 6.Watson CW. Effect of lowering of body temperature on the symptoms and signs of multiple

278 sclerosis. New England Journal of Medicine. 1959;261(25):1253-9.

279 7.White A, Wilson T, Davis S, Petajan J. Effect of precooling on physical performance in multiple 280 sclerosis. Multiple Sclerosis. 2000;6(3):176-80.

281 8.Meyer-Heim A, Rothmaier M, Weder M, Kool J, Schenk P, Kesselring J. Advanced lightweight 282 cooling-garment technology: functional improvements in thermosensitive patients with multiple 283 sclerosis. Multiple Sclerosis. 2007;13(2):232-7.

284 9.Society NMS. Heat and Temperature Sensitivity 2004 [updated December 4, 2015]. Available 285 from: http://www.nationalmssociety.org/Living-Well-With-MS/Health-Wellness/Heat-

286 Temperature-Sensitivity\#section-3.

287 10.Society MS. Hot and Cold2013:[4 p.]. Available from:

288 https://www.mssociety.org.uk/sites/default/files/Documents/Essentials/Hot-and-cold-temperature289 factsheet-Feb-13.pdf. 
290 11.Kurtzke JF. Rating neurologic impairment in multiple sclerosis an expanded disability status 291 scale (EDSS). Neurology. 1983;33(11):1444-.

292 12.Burdon CA, Hoon MW, Johnson NA, Chapman PG, O’Connor HT. The effect of ice slushy

293 ingestion and mouthwash on thermoregulation and endurance performance in the heat.

294 International journal of sport nutrition and exercise metabolism. 2013;23(5):458-69.

295 13.Ramanathan N. A new weighting system for mean surface temperature of the human body.

296 Journal of Applied Physiology. 1964;19(3):531-3.

297 14.Astrand P, Rodahl K. Evaluation of physical work capacity on the basis of tests. Textbook of 298 work physiology: Physiological basis of exercise Human Kinetics1977. p. 333-65.

299 15.Fitchett M. Predictability of VO2 max from submaximal cycle ergometer and bench stepping 300 tests. British journal of sports medicine. 1985;19(2):85-8.

301 16.Uhthoff W. Studies on the occurring in multiple sclerosis stove eye disorders. Arch. f.

302 Psychiatrie u. Nervenheilk Bd XXI. 1889.

303 17.Marino FE. Heat reactions in multiple sclerosis: An overlooked paradigm in the study of 304 comparative fatigue. International Journal of Hyperthermia. 2009;25(1):34-40.

305 18.Tyler CJ, Sunderland C, Cheung SS. The effect of cooling prior to and during exercise on 306 exercise performance and capacity in the heat: a meta-analysis. British journal of sports medicine. 307 2013:bjsports-2012-091739.

308 19.Morris NB, Bain AR, Cramer MN, Jay O. Evidence that transient changes in sudomotor output 309 with cold and warm fluid ingestion are independently modulated by abdominal, but not oral 310 thermoreceptors. Journal of Applied Physiology. 2014;116(8):1088-95.

311 20.Morris NB, Coombs G, Jay O. Ice Slurry Ingestion Leads to a Lower Net Heat Loss during 312 Exercise in the Heat. Medicine and science in sports and exercise. 2016;48(1):114-22.

313 21.Bain A, Lesperance N, Jay O. Body heat storage during physical activity is lower with hot fluid 314 ingestion under conditions that permit full evaporation. Acta Physiologica. 2012;206(2):98-108. 
315 22.Sandroff BM, Dlugonski D, Weikert M, Suh Y, Balantrapu S, Motl RW. Physical activity and 316 multiple sclerosis: new insights regarding inactivity. Acta Neurologica Scandinavica.

317 2012;126(4):256-62. doi: 10.1111/j.1600-0404.2011.01634.x.

318 23.Motl RW, McAuley E, Snook EM. Physical activity and multiple sclerosis: a meta-analysis.

319 Multiple Sclerosis Journal. 2005;11(4):459-63.

320 24.Beenakker E, Oparina T, Hartgring A, Teelken A, Arutjunyan A, De Keyser J. Cooling garment

321 treatment in MS: clinical improvement and decrease in leukocyte NO production. Neurology.

322 2001;57(5):892-4.

323 25.Ely BR, Cheuvront SN, Kenefick RW, Sawka MN. Aerobic performance is degraded, despite

324 modest hyperthermia, in hot environments. Medicine and science in sports and exercise.

325 2010;42(1):135-41. doi: 10.1249/MSS.0b013e3181adb9fb.

326

327 


\section{$328 \quad$ Figure Legends}

329 Figure 1. Individual data and group means (with SD) at the end of exercise in NEU 330 (yellow) trial compared to the same time point in the CLD (black) trial for multiple 331 sclerosis (MS: squares) and healthy controls (CON: circles). Values given for: Exercise 332 time to exhaustion with a maximum of $60 \mathrm{~min}$. Asterisk (*) indicates $\mathrm{P} \leq 0.05$.

333 Figure 2. Individual data and group means (with SD) at the end of exercise in NEU 334 (yellow) trial compared to the same time point in the CLD (black) trial for multiple 335 sclerosis (MS: squares) and healthy controls (CON: circles). Values given for: Rectal 336 temperature (Panels A-B), mean skin temperature (Panel C-D) and heart rate (Panel E-F) 337 Asterisk $(*)$ indicates $\mathrm{P} \leq 0.05$. 
347

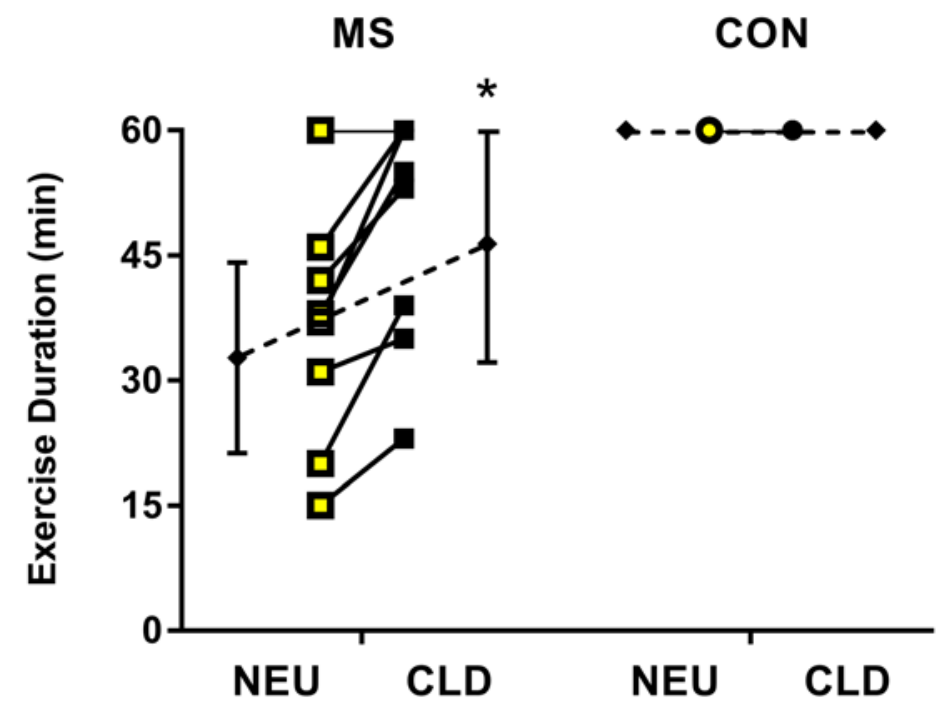

348 Figure 1.

349

350

351

352

353

354

355

356

357 

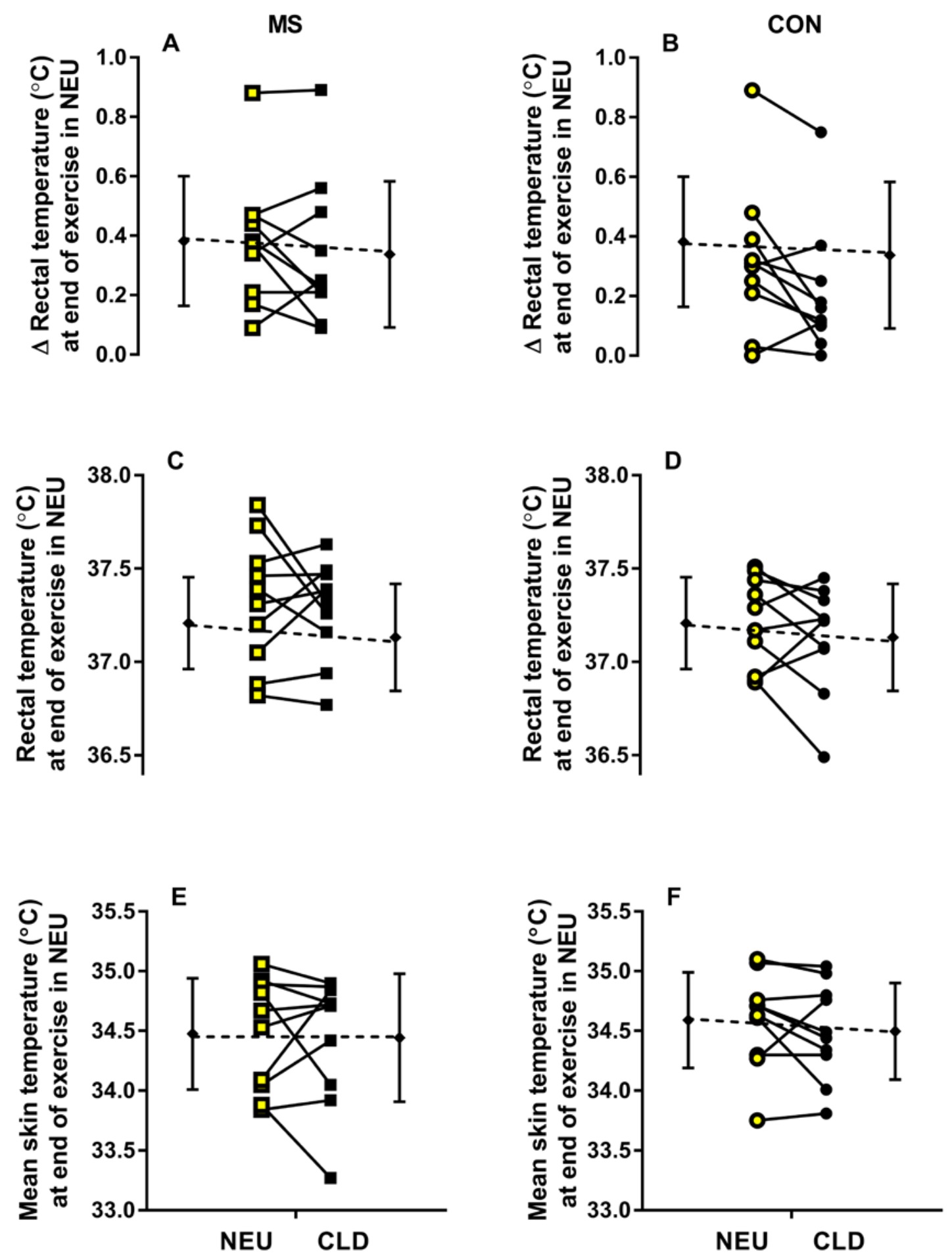

359 Figure 2. 